\title{
Survival following decompressive hemicraniectomy for hemiconvulsion-hemiplegia-epilepsy syndrome: case report
}

\author{
Alexandra D. Beier, DO, ${ }^{1}$ Gemi E. Jannotta, MSN, ARNP, ${ }^{1}$ E. Dayan Sandler, PharmD, MD, ${ }^{2}$ \\ Harry S. Abram, MD, ${ }^{3}$ Raj D. Sheth, MD, ${ }^{3}$ and Philipp R. Aldana, MD ${ }^{1}$ \\ 'Division of Pediatric Neurosurgery, University of Florida Health; 2Jacksonville Pathology Consultants, Chief of Pathology; and \\ 3Department of Neurology, Nemours Children's Specialty Care, Jacksonville, Florida
}

\begin{abstract}
Hemiconvulsion-hemiplegia-epilepsy (HHE) is an uncommon epileptic syndrome that affects young children. Typical management includes early initiation of benzodiazepines to abate the initial seizure activity quickly. Patients in whom epilepsy develops require prolonged use of antiepileptic agents. Herniation due to diffuse cerebral edema from HHE is rare; however, decompressive craniectomy has been described as a lifesaving measure. The authors present the case of a patient in whom a decompressive craniectomy was performed. They advocate a proactive approach in the detection and management of cerebral edema in HHE causing intracranial hypertension. In HHE cases that exhibit radiographic evidence of malignant cerebral edema (although not previously described in this disease, but similar to the setting of stroke and trauma), the authors advocate early neurosurgical consultation and evaluation for insertion of an intracranial pressure monitor for those patients who do not have a reliable neurological examination (i.e., Glasgow Coma Scale score $\leq 8)$.
\end{abstract}

http://thejns.org/doi/abs/10.3171/2016.3.PEDS15677

KEY WORDS HHE; hemicraniectomy; epilepsy; hemiconvulsion-hemiplegia-epilepsy syndrome; technique

$\mathrm{H}$ EMICONVULSION-HEMIPLEGIA-EPILEPSY (HHE) syndrome is an uncommon result of prolonged focal status epilepticus in childhood and was first described by Gastaut et al. in $1960 .^{7}$ With recent medical advancements, the incidence of HHE is perceived to be declining. Medical treatment of associated status epilepticus usually prevails; however, occasionally if the status is intractable, surgical options, including intracranial pressure (ICP) monitoring and decompressive hemicraniectomy, are pursued. ${ }^{2}$ The incidence of HHE resulting in cerebral herniation is not well known but has been described and can result in severe morbidity or even death. To date, there are only a few reported pediatric cases of HHE that have required surgical treatment of cerebral herniation in the form of a decompressive hemicraniectomy. ${ }^{3,9}$ We present a report on the successful use of decompressive hemicraniectomy for the treatment of cerebral herniation in HHE and advocate close monitoring for development of intracranial hypertension in these patients.

\section{Case Report}

A previously healthy 2-year-old girl with a 24-hour history of generalized malaise and fever of $101^{\circ} \mathrm{F}$ presented to the emergency department in prolonged status epilepticus that failed to respond to diazepam and required 3 doses of $0.5 \mathrm{mg}$ of intravenous lorazepam. A loading dose

ABBREVIATIONS EEG = electroencephalography; GCS = Glasgow Coma Scale; HHE = hemiconvulsion-hemiplegia-epilepsy; ICP = intracranial pressure; MRA = MR angiography. 


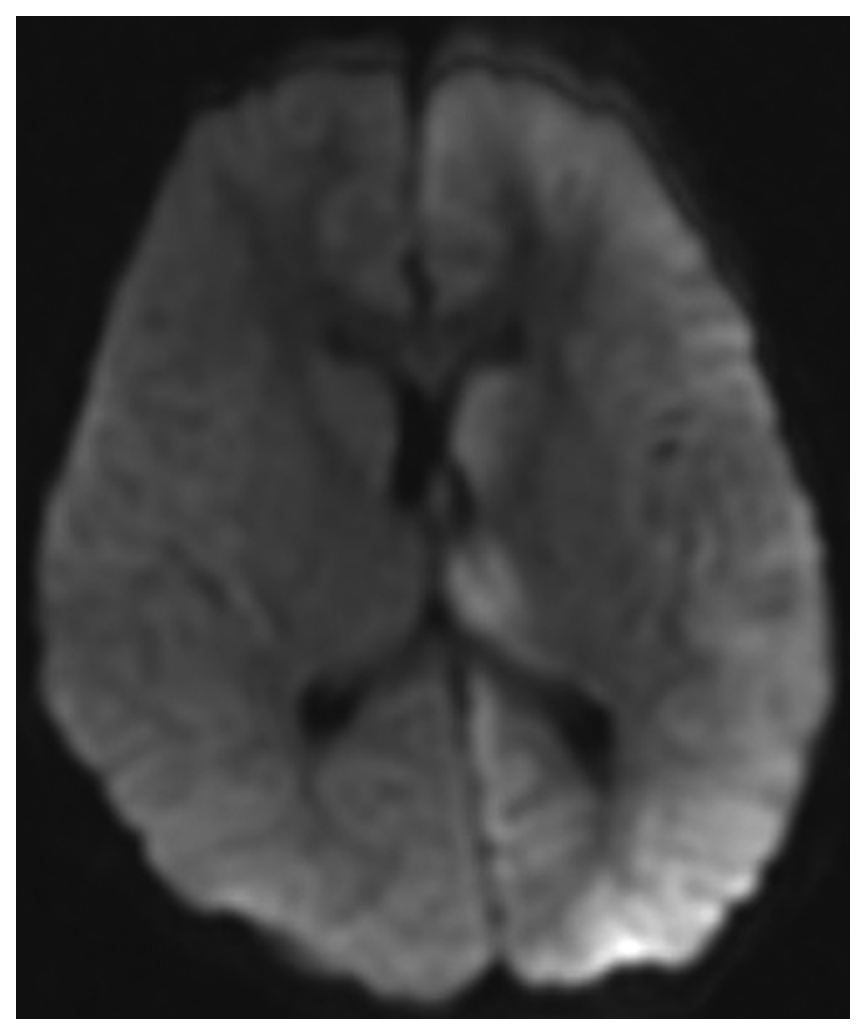

FIG. 1. Axial diffusion-weighted MR image obtained 24 hours after admission.

of fosphenytoin and broad-spectrum intravenous antibiotics were administered, and a lumbar puncture was performed in the emergency department. The resolution of the seizures was asymmetrical, with the left-side twitching resolving 25 minutes prior to the right side remitting. Intermittent orobucal automatism lasting approximately 2 seconds in duration after resolution of the convulsive status was documented. The patient underwent CT scanning 5 hours after admission, the findings of which were normal. The primary treating physicians recommended admission to the pediatric ICU from the emergency department after initial seizure control was obtained with the administration of levetiracetam, lorazepam, fosphenytoin, and phenobarbital.

After she was admitted to the pediatric ICU for observation and continuous electroencephalography (EEG), the patient relapsed into status epilepticus approximately 5 hours after admission. She was intubated for airway protection and was treated with pentobarbital coma for seizure control, which was noted to be effective on EEG performed at approximately 23 hours after admission to the hospital. MRI of the brain at 24 hours after admission was performed and demonstrated extensive left hemispheric restricted diffusion, consistent with changes after status epilepticus (Fig. 1). These MRI findings were consistent with the patient's EEG findings that demonstrated her initial presentation with status epilepticus. MR angiography (MRA) was also performed at 24 hours and revealed increased perfusion of the left cerebral hemisphere and posterior fossa, likely due to status epilepticus, without

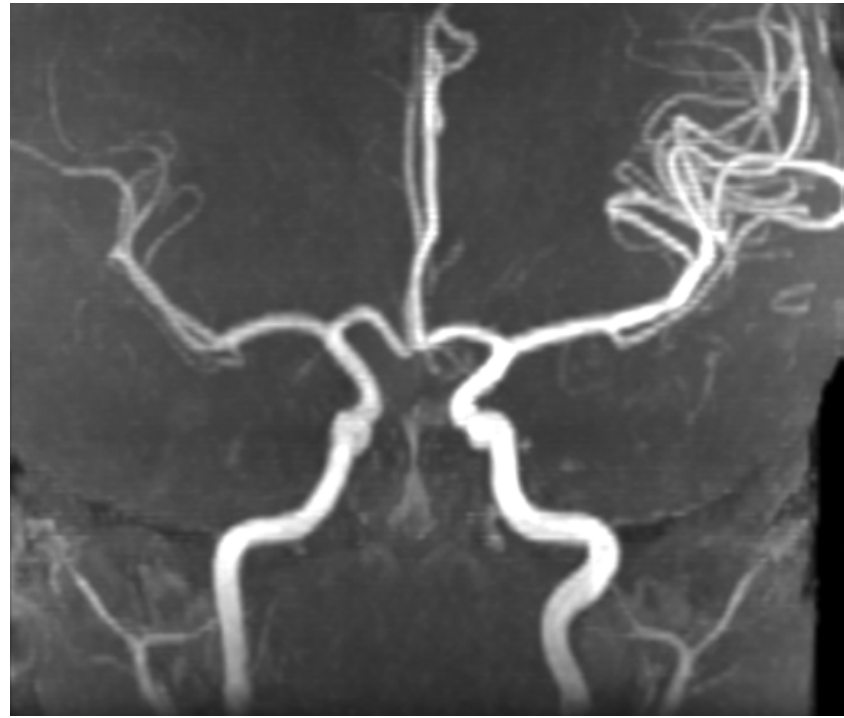

FIG. 2. An MR angiogram revealing no branch stenosis and increased perfusion of the left hemisphere.

evidence of branch stenosis (Fig. 2). The primary treating team managed the patient with continuous EEG, hourly neurological examinations, and the medications noted above. Within the limitation of the patient's clinical examination, secondary to seizure control with multiple antiepileptic agents and the use of pentobarbital, there were no reported changes in her examination findings, and she remained in burst suppression. Approximately 48 hours after her arrival she was noted to have left pupillary dilation. A CT scan obtained immediately revealed a diffuse cerebral edema, with left sulcal effacement, mass effect with midline shift, and uncal herniation (Fig. 3). At that time, an initial neurosurgical evaluation was performed, and the decision was made for an emergent surgical decompression as a lifesaving measure. The patient underwent a left decompressive hemicraniectomy, brain biopsy, insertion of an external ventricular drain and a subdural ICP monitor, and a postoperative head CT scan was obtained (Fig. 4). Photomicrographs of the pathological changes found in the tissue are shown in Fig. 5. This tissue was obtained to evaluate for other pathology, including vasculitis. The cytological changes identified were consistent with anoxia and infarction, and no evidence of vasculitis, infection, or tumor was found.

In the immediate postoperative timeframe, the patient's ICP was well controlled at 6-7 mm Hg. She did not manifest intracranial hypertension during her postoperative course. She remained in the ICU for 2 weeks, where the ventilator and external ventricular drain were weaned and the ICP monitor was discontinued. Neurologically she exhibited right hemiplegia with marked spasticity, but she remained seizure free. She continued receiving phenobarbital and levetiracetam for seizure prevention. No viral elements (such as herpes simplex virus or human immunodeficiency virus [HIV]) or bacterial agents were isolated after clinical and biological analysis. She was transferred to an inpatient rehabilitation facility after approximately 30 inpatient days. At 3 months post-hemicraniectomy, the 


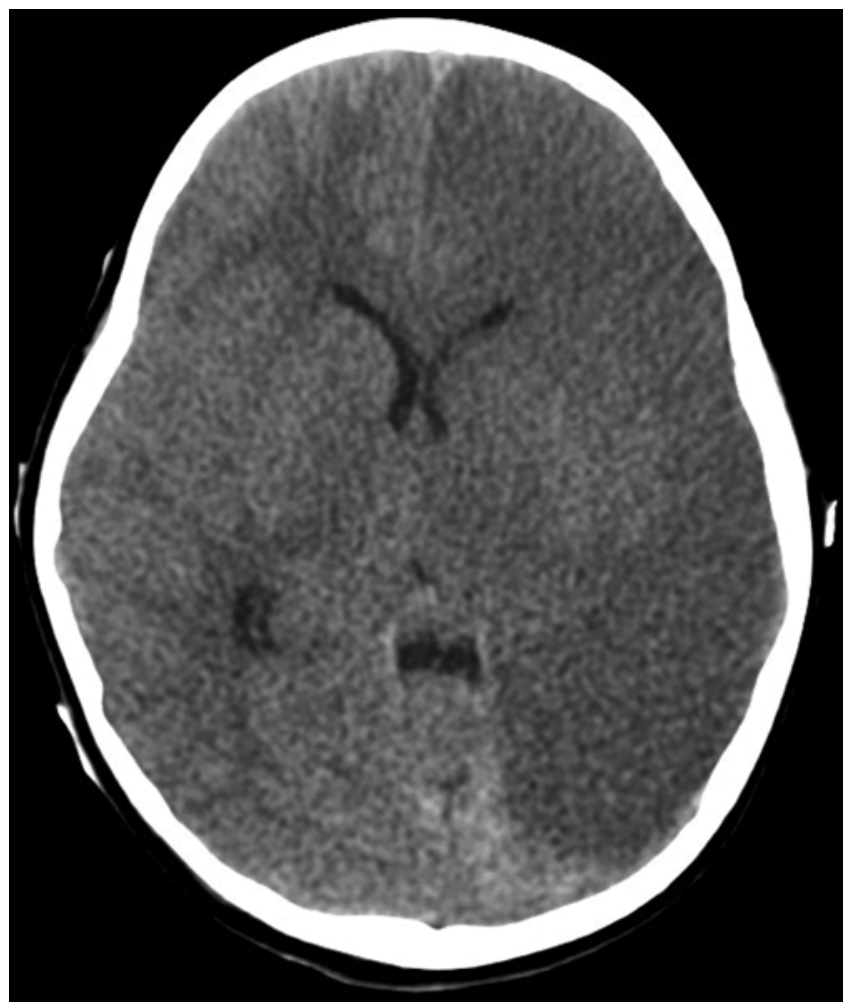

FIG. 3. Axial CT scan obtained after pupillary change, demonstrating left hemispheric edema.

patient underwent reimplantation of her skull flap, with subsequent partial resorption requiring revision. Neurologically she continues to have right-sided hypertonicity and hemiparesis, with the upper extremity more affected than the lower; however, she is able to ambulate and communicate verbally. At last follow-up (48 months after surgery) she was making significant cognitive progress. With regard to her seizures, she continued receiving phenobarbital for approximately 1 year post-decompression and was successfully weaned. She remains seizure free, receiving oxcarbazepine as her only medication.

\section{Discussion}

HHE is a prolonged unilateral febrile seizure resulting in permanent hemiparesis and is seen in children younger than 4 years. The pathological substrate involves cerebral edema restricted to the affected hemisphere. Late results of HHE are a progressive hemiatrophy of one cerebral hemisphere. ${ }^{14}$ The etiology of HHE remains poorly defined, although it can be related to a focal cortical malformation of the dyslamination type (focal cortical dysplasia, Type 1), which is frequently associated with MRI-negative epilepsy. ${ }^{6}$ Other causes of HHE include infectious, vascular, and metabolic processes, or it can be idiopathic. ${ }^{11}$ Generally, the HHE syndrome progresses insidiously to clonic partial epilepsy. Most cases do not result in cerebral herniation, but the incidence of this is not well known. Occasionally, acute, life-threatening presentations can occur, as evidenced by a previously discussed case. ${ }^{10}$

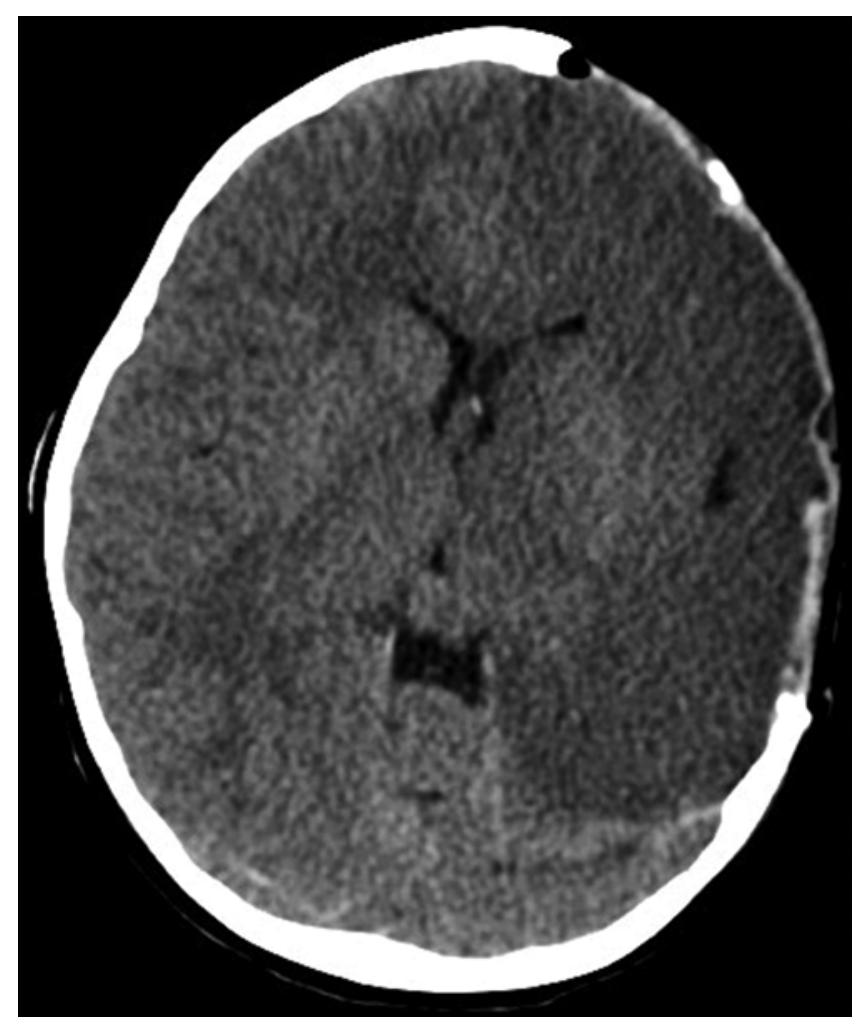

FIG. 4. Axial CT scan obtained after hemicraniectomy, revealing postoperative changes.

Decompressive hemicraniectomy is an acute surgical intervention used to prevent the progression of cerebral herniation acutely or to help control intracranial hypertension that is refractory to medical therapy. Traditionally, etiologies that benefit from a hemicraniectomy include massive unilateral ischemic stroke, dural sinus thrombosis, intracerebral hematoma, traumatic brain injuries (especially subdural hematoma), encephalitis, subdural empyema, and aneurysmal subarachnoid hemorrhages. The common pathological aspects of these diseases involve significant cerebral edema and elevated ICP..$^{13}$ The overall goal of the hemicraniectomy is to increase the volumebuffering capacity of the cranial vault, allowing extracranial supratentorial herniation to diminish the pressure gradients that cause cerebral herniation. The overarching benefit of early hemicraniectomy is that uncontrollable increased ICP can be thwarted prior to loss of autoregulation, thereby reducing overall mortality. ${ }^{8}$ Unfortunately, the neurocognitive sequela secondary to increased ICP exists on a continuum of severity, and it is nearly impossible to determine the quality of life a patient might experience after a hemicraniectomy. Many believe the cons of early hemicraniectomy are centered on these perceived ideas of a poor quality of life after recovery. According to Jagannathan et al., ${ }^{8}$ in evaluating decompressive craniectomies for traumatic brain injuries in pediatric patients, they found that a decompressive craniectomy is associated with favorable outcomes. The authors further described that the rationale for more aggressive surgical treatment in pediatric patients is warranted because children have been 


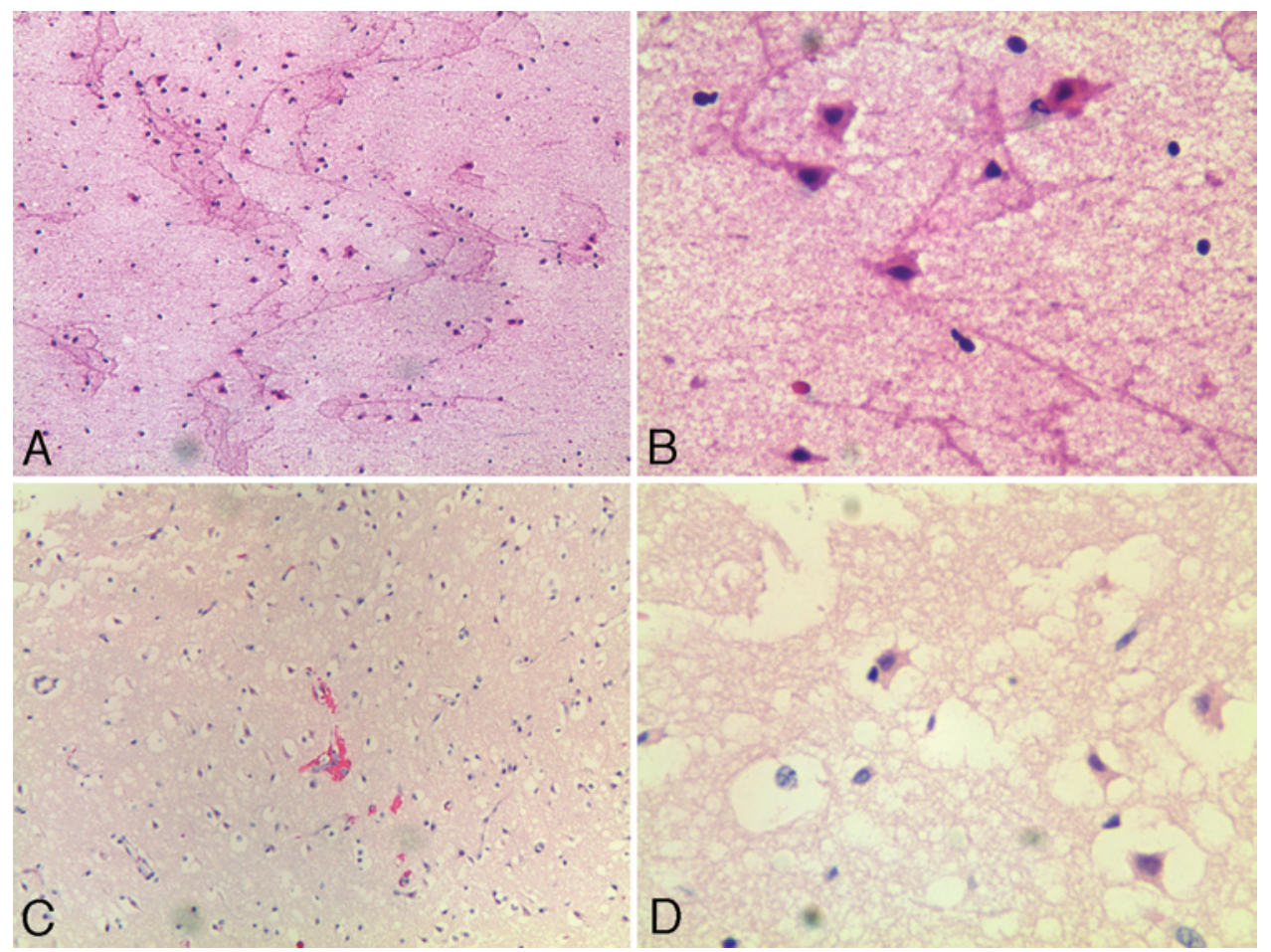

FIG. 5. A: Low-power field of the smear from the submitted biopsy sample showing hyperchromatic nuclei of neural cells suggestive of anoxic effects. B: At high power, the neurons show intense eosinophilia of the cytoplasm, "red neurons," and shrinkage of the cell bodies. The nuclei show pyknosis, as a result of the condensation of the chromatid. C: This low-power view of the tissue section shows extensive vacuolar change around neurons with condensed nuclei. Nucleoli, seen in normal neurons, are not evident here. Small capillaries show vascular congestion. D: At high power, this section shows extensive vacuolar change around neurons and spongiosis of brain parenchyma. The neurons show significant anoxic effect, characterized by loss of nucleoli and extensive pyknosis. Diff-Quick Stains ( $A$ and $B)$ and $H$ \& E (C and D), original magnification $\times 10$ ( $A$ and C), x100 (B), and ×60 (D). Figure is available in color online only.

found to show 5 times as much cerebral edema when compared with adults in the setting of traumatic brain injuries, and thus they benefit from early intervention. ${ }^{8}$

Decompressive hemicraniectomy in the management of HHE causing malignant cerebral edema and cerebral herniation has been reported only a few times in the literature. Berhouma et al. described the case of a previously healthy child younger than 4 years who presented with fever and prolonged status epilepticus with unwitnessed initial seizure onset. The patient required a decompressive hemicraniectomy due to cerebral herniation. ${ }^{3}$ Jayakody and Joshi ${ }^{9}$ also described the case of a previously healthy 3 -year-old child who presented with left focal motor status epilepticus with unwitnessed initial seizure onset, who suffered cardiac arrest during her hemicraniectomy due to cerebral herniation and ultimately did not survive. In this patient, autopsy revealed marked asymmetrical edema of the affected hemisphere. Pathological examination revealed neuronal shrinkage, cytoplasmic eosinophilia, and nuclear pyknosis, consistent with pathological findings in our case. ${ }^{9}$ Similar to our case, both of these patients were initially treated with anticonvulsant medications and experienced prolonged status epilepticus that was difficult to abate. Also, each child experienced neurological decline resulting in pupillary dilation and herniation. It is interesting to note that the time of onset for the pupillary change for each patient was within 36-72 hours from the onset of fever and illness, including reported prehospital febrile illness. In the case of Berhouma et al., the patient was treated with antiepileptic medications, intubated, and sent directly for cranial imaging due to the pupillary change noted on admission. Ultimately the patient underwent emergent right hemicraniectomy. ${ }^{3}$ In the patient described by Jayakody and Joshi, a head CT was obtained on admission that revealed right hemispheric edema and a possible left small subdural hematoma, and thus the patient was transferred to a larger facility for further treatment. ${ }^{9}$ Findings on a repeat CT scan obtained 2.5 hours later were unchanged, and brain MRI performed 4 hours later showed right hemispheric restricted diffusion with gyral edema involving 3 vascular territories with normal MRA findings of the head and neck. This patient's pupillary change was noted directly following attenuation of all EEG activity (about 31 hours after initial presentation) and this prompted repeat imaging that led to emergent right hemicraniectomy. ${ }^{3,9}$ In the current case, the patient also underwent imaging on admission, as well as 24 hours after admission; however, there was no evidence of herniation on those evaluations. The patient underwent imaging again directly following the pupillary change, 48 hours after admission, and herniation was confirmed. These 3 case studies provide evidence to support that frequent imaging 
is warranted to identify impending herniation before clinical symptoms arise, as each patient had significant decline within 48 hours of admission.

A consideration must be made for cerebrovascular accident as the cause for a child's neurological condition when one presents with seizures, especially when there are diffusion changes present on imaging. Abend and Marsh found that seizures were the presenting symptoms of acute arterial ischemic stroke in $22 \%$ of their studied pediatric population. ${ }^{1}$ Singh et al. reported similarly that clinical seizure activity was the initial sign of stroke in $21 \%$ of pediatric patients being evaluated for a first symptomatic stroke. ${ }^{12}$ However, Abend and Marsh further detailed that all patients presenting with seizures in their series also had hemiparesis present on admission. ${ }^{1}$ With regard to the current case, the patient's examination was limited upon arrival due to the multiple antiepileptic medications. Yet the MRA ruled out branch occlusion that could account for the diffusion-weighted changes on MRI. Of note, Cartagena et al. described reversible and irreversible MRI findings seen in status epilepticus. These changes range from diffusion changes, alterations in apparent diffusion coefficient values, and T2 hyperintensities, with locations varying from unilateral focal lesions to bilateral lesions to hemispheric involvement. ${ }^{5}$ Therefore, cerebrovascular accidents should be part of the differential diagnosis for children presenting with seizures, and a stroke evaluation should be considered as well as a vascular evaluation to rule out stroke as a cause for the MRI changes, understanding that these changes can also be seen with status epilepticus.

Due to the natural history of cerebral herniation syndrome and the urgency with which surgical intervention is required when signs of herniation occur, it could be theorized from these cases along with traumatic brain injury data and guidelines that a systematic approach to detecting and monitoring and treating patients with HHE who develop malignant cerebral edema is warranted. ${ }^{8}$ In the patient who develops signs of cerebral herniation in the setting of HHE, we advocate emergent decompressive hemicraniectomy as a lifesaving measure, as these 3 cases illustrate. All patients with HHE should be aggressively treated to cease epileptiform activity, also realizing that sudden abatement can also be a sign of herniation. In those HHE cases that exhibit radiographic evidence of malignant cerebral edema, although not previously described in this disease but, similar to the setting of stroke and trauma, we advocate that early neurosurgical consultation for evaluation and consideration for insertion of an ICP monitor should be considered in those patients who do not have a reliable neurological examination (i.e., Glasgow Coma Scale [GCS] score $\leq 8$ ).

The neurological examination findings can be obscured in these patients either due to seizures or to the medical treatment of HHE. This is the precise patient population in which progressive cerebral edema and intracranial hypertension can be easily missed and who can later present with signs of cerebral herniation. In the traumatic brain injury population, patients with a GCS score $\leq 8$ who have abnormal brain imaging findings are recommended to receive an ICP monitor. ${ }^{4}$ Similarly, we advocate that pa- tients with HHE who require medical treatment of their seizures that prohibits a reliable neurological examination for a prolonged period of time (24-48 hours) should be monitored with more frequent brain imaging studies and elective placement of an ICP monitor as discerned by the neurologist and neurosurgeon. Proactive medical management of intracranial hypertension in these patients may mitigate the course of cerebral edema and decrease the need for emergent decompressive hemicraniectomy, thus resulting in improved neurological outcomes.

\section{Conclusions}

HHE is an unfortunate outcome of status epilepticus that can result in significant neurological morbidity and mortality. Proper seizure control is paramount, not only when seizures are visually witnessed, but also during nonconvulsive status epilepticus. Malignant cerebral edema with resultant intracranial hypertension can develop in these patients, and clinicians need to take a proactive approach in its detection and treatment. With radiographic evidence of cerebral edema and an unreliable neurological examination, we advocate insertion of an ICP monitor and institution of medical management to treat intracranial hypertension if present. In those cases with refractory intracranial hypertension or those exhibiting signs of cerebral herniation, a decompressive hemicraniectomy may be necessary. As illustrated in the current case, a decompressive hemicraniectomy in the setting of cerebral herniation in HHE in a child can be lifesaving and result in acceptable neurological outcomes.

\section{References}

1. Abend NS, Marsh E: Convulsive and nonconvulsive status epilepticus in children. Curr Treat Options Neurol 11:262272, 2009

2. Auvin S, Bellavoine V, Merdariu D, Delanoë C, ElmalehBergés M, Gressens P, et al: Hemiconvulsion-hemiplegiaepilepsy syndrome: current understandings. Eur J Paediatr Neurol 16:413-421, 2012

3. Berhouma M, Chekili R, Brini I, Kchir N, Jemel H, Bousnina $\mathrm{S}$, et al: Decompressive hemicraniectomy in a space-occupying presentation of hemiconvulsion-hemiplegia-epilepsy syndrome. Clin Neurol Neurosurg 109:914-917, 2007

4. Bullock RM, Chesnut RM, Clifton GL, Ghajar J, Marion DW, Naryan RK, et al: Guidelines for the management of severe head injury. Indications for intracranial pressure monitoring. J Neurotrauma 17:479-491, 2000

5. Cartagena AM, Young GB, Lee DH, Mirsattari SM: Reversible and irreversible cranial MRI findings associated with status epilepticus. Epilepsy Behav 33:24-30, 2014

6. Franzoni E, Garone C, Marchiani V, Brunetto D, Tonon C, Lodi R, et al: A new case of idiopathic hemiplegia hemiconvulsion syndrome. Neurol Sci 31:799-805, 2010

7. Gastaut H, Poirier F, Payan H, Salamon G, Toga M, Vigouroux M: H.H.E. syndrome; hemiconvulsions, hemiplegia, epilepsy. Epilepsia 1:418-447, 1960

8. Jagannathan J, Okonkwo DO, Yeoh HK, Dumont AS, Saulle D, Haizlip J, et al: Long-term outcomes and prognostic factors in pediatric patients with severe traumatic brain injury and elevated intracranial pressure. J Neurosurg Pediatr 2:240-249, 2008

9. Jayakody H, Joshi C: Fatal outcome in hemiconvulsionhemiplegia syndrome. J Child Neurol 29:406-411, 2014 
10. Mori Y: Anatomopathology and pathogeny of the hemiconvulsion-hemiplegia-epilepsy syndrome. Part II. J Neurosurg Sci 23:1-22, 1979

11. Salih MA, Kabiraj M, Al-Jarallah AS, El Desouki M, Othman S, Palkar VA: Hemiconvulsion-hemiplegia-epilepsy syndrome. A clinical, electroencephalographic and neuroradiological study. Childs Nerv Syst 13:257-263, 1997

12. Singh RK, Zecavati N, Singh J, Kaulas H, Nelson KB, Dean NP, et al: Seizures in acute childhood stroke. J Pediatr 160:291-296, 2012

13. Stan H, Suciu B, Iosif A, Cocis A: Consideration on hemicraniectomy. Romanian Neurosurgery 16:37-42, 2000

14. Tenney JR, Schapiro MB: Child neurology: hemiconvulsionhemiplegia-epilepsy syndrome. Neurology 79:e1-e4, 2012

\section{Disclosures}

The authors report no conflict of interest concerning the materi- als or methods used in this study or the findings specified in this paper.

\section{Author Contributions}

Conception and design: Aldana. Acquisition of data: Jannotta, Abram. Analysis and interpretation of data: Sandler, Aldana. Drafting the article: Beier, Jannotta. Critically revising the article: Beier, Jannotta, Abram, Aldana. Reviewed submitted version of manuscript: all authors. Approved the final version of the manuscript on behalf of all authors: Beier. Administrative/ technical/material support: Jannotta. Study supervision: Aldana.

\section{Correspondence}

Alexandra D. Beier, Division of Pediatric Neurosurgery, University of Florida Health Jacksonville, 836 Prudential Dr., Ste. 1205, Jacksonville, FL 32207. email: alexandra.beier@ jax. ufl.edu. 